\title{
State-Feedback Control for Fractional-Order Nonlinear Systems Subject to Input Saturation
}

\author{
Junhai Luo \\ School of Electronic Engineering, University of Electronic Science and Technology of China, Chengdu 611731, China \\ Correspondence should be addressed to Junhai Luo; junhai_luo@uestc.edu.cn \\ Received 23 September 2013; Revised 22 December 2013; Accepted 24 December 2013; Published 12 January 2014 \\ Academic Editor: Yuncai Wang \\ Copyright (C) 2014 Junhai Luo. This is an open access article distributed under the Creative Commons Attribution License, which \\ permits unrestricted use, distribution, and reproduction in any medium, provided the original work is properly cited. \\ We give a state-feedback control method for fractional-order nonlinear systems subject to input saturation. First, a sufficient \\ condition is derived for the asymptotical stability of a class of fractional-order nonlinear systems. Then based on Gronwall-Bellman \\ lemma and a sector bounded condition of the saturation function, a linear state-feed back controller is designed. Finally, two \\ simulation examples are presented to show the validity of the proposed method.
}

\section{Introduction}

The idea of fractional calculus has been proposed since the development of the integer order calculus, with the initial works of Leibniz and L'Hospital. In spite of being such an old topic, the developments in this field were rather slow. However, in the last two decades, fractional differential equations have been utilized more and more to model various physical phenomena. In fact, recent developments of fractional calculus are dominated by modern examples of applications in differential and integral equations, physics, fluid mechanics, signal processing, mathematical biology, viscoelasticity, electrochemistry, and many others. And we can refer to [1] for the recent history of fractional calculus. Fractional calculus is becoming a new mathematical method of solution of diverse problems in mathematics, science, and engineering [2-5].

As a result of the growing applications, the study of stability of fractional differential equations has attracted much attention. The earliest discussion on stability of fractional differential equations (FDEs) can be traced back to Matignon [6], where it was known that the stability problem of FDEs comes down to the eigenvalue problem of system matrices. For fractional-order linear time-invariant interval systems, the stability problems were discussed in [7-10]. In [7], a stability criterion which was based on the Lyapunov inequality was given. The necessary and sufficient conditions based on the linear matrix inequalities methods with fractional-order $0<\alpha<1$ were derived in [8], and $1 \leq \alpha<2$ in $[9,10]$, respectively. In $[11,12]$, the stability of the perturbed fractional-order linear systems is investigated in the general case. In [13], the asymptotically stabilization of fractional-order linear systems subject to input saturation is investigated. In [14], both state and output feedback stabilization controllers are designed for triangular fractional-order linear time-invariant systems with fractional-order $0 \leq \alpha<2$.

On the other hand, because fractional differential operators are nonlocal and have weakly singular kernels, some methods in handling integer-order systems cannot be simply extended to fractional-order systems. To the best of our knowledge, there are only several results on the stability of fractional-order nonlinear systems. For example, the definition of Mittag-Leffler stability and the fractional Lyapunov direct method to discuss the stability of fractional-order nonlinear dynamic systems are proposed in $[15,16]$. Sadati et al. give two theorems for the Mittag-Leffler stability of fractional nonlinear systems with time delay [17]. Deng provides sufficient conditions for locally asymptotical stability of nonlinear fractional-order differential equations with fractional-order $\alpha$ : $0<\alpha<1$ [18]. In [19], the stability and stabilization of a class of fractional-order nonlinear systems with Caputo derivative are studied with fractionalorder $\alpha$ : $0<\alpha<1$ and $1<\alpha<2$. 
All real world technical systems are subjected to input constraints. In many engineered systems, input saturation does exist due to a limited size of sensors, actuators, and some interfacing devices. The existence of input saturation may decrease the control performance or cause oscillations and even lead to instability of the system [13, 20]. For the integer-order linear and nonlinear systems, input saturation has received much attention from researchers in the past decade. The sector bounded condition associated with input nonlinearities is useful for analysis and synthesis of control systems subject to input saturation. Then the stability of the system can be formulated using Lyapunov stability theory and invariant theory.

Though significant research efforts have been put to the fractional-order time-invariant systems, fractional-order systems subject to input saturation have rarely been investigated in the literature. Here, with the help of the Laplace transform, Mittag-Leffler function, and Gronwall inequality, a statefeedback controller is designed for a class of fractional-order nonlinear systems subject to input saturation. Compared with $[12,13,19]$, there are some main contributions that are worth to be emphasized.

(1) A sufficient condition is proposed for the asymptotical stability and stabilization of fractional-order chaotic systems subject to input saturation with fractional order $0<\alpha<1$.

(2) A linear state-feedback controller is constructed to stabilize the fractional-order chaotic systems. It is shown that linear controller can also be used to control fractional-order nonlinear systems (chaotic or nonchaotic).

(3) In this paper, we assume that the nonlinear function $f(x(t))$ is locally Lipschitz in $x$. Compared with our assumption, restrictive assumptions are made on $f(x(t))$ in $[12,19]$, namely,

$$
\lim _{x(t) \rightarrow 0} \frac{\|f(x(t))\|}{\|x(t)\|}=0 .
$$

\section{Preliminaries}

The Caputo definition of fractional-order derivatives can be written as [4]

$$
\begin{array}{r}
{ }_{0}^{C} D_{t}^{\alpha} x(t)=\frac{1}{\Gamma(n-\alpha)} \int_{0}^{t} \frac{x^{(n)}(\tau)}{(t-\tau)^{\alpha-n+1}} d \tau, \\
n-1<\alpha<n,
\end{array}
$$

where $\alpha$ is the fractional order, and the gamma function $\Gamma(\cdot)$ is defined as $\Gamma(\tau)=\int_{0}^{\infty} t^{\tau-1} e^{-t} d \tau$.

The Laplace transform of Caputo fractional derivative can be given as follows:

$$
\int_{0}^{\infty} e^{-s t}{ }_{0}^{C} D_{t}^{\alpha} x(t) d t=s^{\alpha}-\sum_{k=0}^{n-1} s^{\alpha-k-1} x^{(k)}(0) .
$$

Throughout this paper, the following definition and lemmas will be used.
Definition 1. The Mittag-Leffler function with two parameters can be written as

$$
E_{\alpha, \beta}(z)=\sum_{k=0}^{\infty} \frac{z^{k}}{\Gamma(\alpha k+\beta)},
$$

where $\alpha, \beta>0$ and $z \in C$.

The Laplace transform of Mittag-Leffler function can be given as

$$
\mathscr{L}\left\{t^{\beta-1} E_{\alpha, \beta}\left(-\lambda t^{\alpha}\right)\right\}=\frac{s^{\alpha-\beta}}{s^{\alpha}+\lambda} .
$$

Lemma 2 (see [12]). If $A \in R^{n \times n}, 0<\alpha \leq 1, \beta$ is an arbitrary real number, and $C>0$ is a real constant, then

$$
E_{\alpha, \beta}(A) \leq \frac{C}{1+\|A\|},
$$

where $\mu \leq|\arg (\operatorname{eig}(A))| \leq \pi$ with $\mu \in R$ satisfies $\pi \alpha / 2<\mu<$ $\min \{\pi, \pi \alpha\}$.

Lemma 3 (see [19]). Ift $\in[0, T]$ and

$$
x(t) \leq h(t)+\int_{0}^{t} k(\tau) x(\tau) d \tau
$$

where $k(t) \geq 0$ and all the functions involved are continuous on the interval $[0, T]$, then we can obtain

$$
x(t) \leq h(t)+\int_{0}^{t} k(\tau) h(\tau) \exp \left[\int_{\tau}^{t} k(u) d u\right] d \tau .
$$

\section{Stability Analysis and State-Feedback Controller Design}

In this section, a sufficient condition is given for the asymptotical stability and stabilization for a class of fractional-order nonlinear systems with input saturation.

3.1. Stability of Fractional-Order Nonlinear System. Let us consider the following fractional-order nonlinear system:

$$
{ }_{0}^{C} D_{t}^{\alpha} x(t)=A x(t)+B f(x(t)), \quad x(0)=x_{0},
$$

where $x(t), f(x(t)), u(t) \in R^{n}$ are the state, nonlinear function, and control input, respectively. $A, B$ are constant matrices of appropriate dimensions, $0<\alpha<1$.

Assumption 4. The nonlinear function $f:[0,+\infty) \times \Omega$ is piecewise continuous in $t$ and locally Lipchitz in $x(t)$ on [0, $+\infty) \times \Omega$ with Lipschitz constant $l$, and $\Omega \in R^{n}$ is a domain that contains the origin $x=0$.

The equilibrium point of system (9) is defined as follows.

Definition 5. The constant $x\left(t_{1}\right)$ is an equilibrium of system (9), if and only if $f\left(x\left(t_{1}\right)\right)=0$.

In this paper, let the equilibrium point be $x=0$. 
Remark 6. For convenience, we state all theorems for the case when the equilibrium point is $x=0$. There is no loss of generality in doing so because any equilibrium point can be moved to the origin via a change of system variables. Suppose that the equilibrium point is $x\left(t_{2}\right) \neq 0$. Consider the change of variable $y(t)=x(t)-x\left(t_{2}\right)$. Then

$$
{ }_{0}^{C} D_{t}^{\alpha} y(t)={ }_{0}^{C} D_{t}^{\alpha}\left(x(t)-x\left(t_{2}\right)\right)=g(y(t)),
$$

and the new system has equilibrium at the origin.

Remark 7. Assumption 4 is not restrictive. From Assumption 4 we know that the nonlinear function $f(x(t))$ satisfies

$$
\|f(x(t))\| \leq l\|x(t)\| .
$$

Compared with our assumption, restrictive assumptions are made on $f(x(t))$ in $[12,19]$, namely,

$$
\lim _{x(t) \rightarrow 0} \frac{\|f(x(t))\|}{\|x(t)\|}=0 .
$$

Note that if (12) is satisfied, there exists some $\delta>0(\delta>0$ may be very small); when $\|x(t)\|<\delta$, we can obtain (11).

Now we are ready to give the following results.

Theorem 8. Consider the fractional dynamic system (9). Suppose that Assumption 4 is satisfied. If $|\arg (\operatorname{eig}(A))|>\alpha \pi /$ 2 and $\alpha\|A\|>c l$, where $c>0$ will be defined later, then system (9) is asymptotically stable.

Proof. By taking the Laplace transform on system (9), we have

$$
s^{\alpha} X(s)=s^{\alpha-1} x_{0}+A X(s)+B \mathscr{L}\{f(x)\},
$$

where $X(s)$ is the Laplace transform of $x(t)$. Let $I$ denote the $n \times n$ identity matrix; we can obtain

$$
X(s)=\left(I s^{\alpha}-A\right)^{-1}\left(s^{\alpha-1} x_{0}+B \mathscr{L}\{f(x)\}\right) .
$$

Taking the Laplace inverse transform on (14), we have the solution of (9):

$$
\begin{aligned}
x(t)= & E_{\alpha, 1}\left(A(t)^{\alpha}\right) x_{0} \\
& +\int_{0}^{t}(t-\tau)^{\alpha-1} E_{\alpha, \alpha}\left(A(t-\tau)^{\alpha}\right) f(x) d \tau .
\end{aligned}
$$

According to Lemma 2, there exist some constants $c>0$ such that

$$
\begin{aligned}
\|x(t)\| \leq & \frac{c\left\|x_{0}\right\|}{1+\|A\| t^{\alpha}} \\
& +\int_{0}^{t} \frac{(t-\tau)^{\alpha-1} c}{1+\|A\|(t-\tau)^{\alpha}}\|f(x)\| d \tau .
\end{aligned}
$$

Based on the properties of Assumption 4 we know that

$$
\|f(x(t))\| \leq l\|x(t)\| .
$$

Then (21) can be rewritten as

$$
\begin{aligned}
\|x(t)\| \leq & \frac{c\left\|x_{0}\right\|}{1+\|A\| t^{\alpha}} \\
& +\int_{0}^{t} \frac{(t-\tau)^{\alpha-1} c l}{1+\|A\|(t-\tau)^{\alpha}}\|x(t)\| d \tau .
\end{aligned}
$$

By using Lemma 3, we have

$$
\begin{aligned}
\|x(t)\| \leq & \frac{c\left\|x_{0}\right\|}{1+\|A\| t^{\alpha}} \\
& +\int_{0}^{t} \frac{c l(t-\tau)^{\alpha-1}\left\|x_{0}\right\|}{\left(1+\|A\|(t-\tau)^{\alpha}\right)\left(1+\|A\| \tau^{\alpha}\right)} \\
= & \frac{c\left\|x_{0}\right\|}{1+\|A\| t^{\alpha}}\left(\int_{\tau}^{t} \frac{c l(t-y)^{\alpha-1}}{1+\|A\|(t-y)^{\alpha}} d y\right) d \tau \\
& +\int_{0}^{t} \frac{c l(t-\tau)^{\alpha-1}\left\|x_{0}\right\|}{\left(1+\|A\| \tau^{\alpha}\right)\left(1+\|A\|(t-\tau)^{\alpha}\right)^{1-(c / \alpha\|A\|)}} d \tau \\
\leq & \frac{c\left\|x_{0}\right\|}{1+\|A\| t^{\alpha}}+c l\left\|x_{0}\right\|\|A\|^{(c l / \alpha\|A\|)-2} \\
& \times \int_{0}^{t}(t-\tau)^{(c l /\|A\|)-1} \tau^{-\alpha} d \tau \\
= & \frac{c\left\|x_{0}\right\|}{1+\|A\| t^{\alpha}}+c l\left\|x_{0}\right\|\|A\|^{(c l / \alpha\|A\|)-2} \\
\Gamma(1+(c l /\|A\|) \Gamma(1-\alpha) & t^{(c l /\|A\|)-\alpha} \\
&
\end{aligned}
$$

Since $\alpha\|A\|>c l$, from (19), we can conclude that

$$
\lim _{t \rightarrow \infty}\|x(t)\|=0,
$$

and this ends the proof.

Remark 9. In $[12,19]$, the authors assume that the nonlinear function $f(x(t))$ satisfies (12). Then there exists some $\delta>$ 0 , such that $\|f(x(t))\| \leq(1 / c)\|x(t)\|$. Thus, the GronwallBellman lemma (Lemma 3) can be used in the stability analysis. In fact, this assumption is very restricted because the $\delta$ may be very small (at the very least, $\delta<1$ ); thus, the results of $[12,19]$ can be used only when the system variables are kept in the small neighbourhood of the origin.

Remark 10. If the nonlinear function $f(x(t))$ is differentiable, the Lipschitz constant $l$ can often be replaced by the bounded derivative of $f(x(t))$; that is, $l=\sup _{t \geq 0}\left(\left\|f^{\prime}(x(t))\right\|\right)$. Subject to Input Saturation. Now let us consider the following 
controlled fractional-order chaotic system subject to input saturation with $0<\alpha<1$ :

$$
{ }_{0}^{C} D_{t}^{\alpha} x(t)=\widetilde{A} x(t)+B f(x(t))+B \text { sat }(u(t)), \quad x(0)=x_{0},
$$

where $x(t), f(x(t)), u(t) \in R^{n}$ are the state, nonlinear function, and control input, respectively. $\widetilde{A}, B$ are constant matrices of appropriate dimensions. $\operatorname{sat}(u)=\left[\operatorname{sat}\left(u_{1}\right), \ldots\right.$, $\left.\operatorname{sat}\left(u_{n}\right)\right]^{T}$ is the vector-valued saturation function with

$$
\operatorname{sat}\left(u_{i}\right)=\operatorname{sign}\left(u_{i}\right) \min \left(u_{0 i},\left|u_{i}\right|\right), \quad i=1,2, \ldots, n,
$$

where $u_{0 i}$ represents the symmetric saturation level relative to the $i$ th control input. input

In this paper, we construct the state-feedback control

$$
u(t)=K x(t)
$$

where $K \in R^{n \times n}$ is constant control gain matrix. Then the closed-loop system of (21) can be rewritten as

$$
\begin{array}{r}
{ }_{0}^{C} D_{t}^{\alpha} x(t)=\widetilde{A} x(t)+B f(x(t))+B \text { sat }( \\
x x(t)), \\
x(0)=x_{0} .
\end{array}
$$

Let us consider the notion of the input saturation condition for memoryless nonlinearities.

Definition 11. A memoryless nonlinearity $\varphi(t, x):[0, \infty) \times$ $R^{p} \rightarrow R^{p}$ is said to satisfy a sector condition if the following inequality holds:

$$
\left(\varphi(t, x)-K_{1} x\right)^{T}\left(\varphi(t, x)-K_{2} x\right) \leq 0, \quad \forall x \in S,
$$

for constant matrices $K_{1}$ and $K_{2}$, where $K_{2}-K_{1}$ is a symmetric positive matrix and $S$ contains the origin.

Based on Definition 11, the following lemma holds:

Lemma 12 (see [21, 22]). Let

$$
S\left(L K, u_{0}\right)=\left\{x(t) \in R^{n} \mid-u_{0} \leq L K x(t) \leq u_{0}\right\},
$$

where $L=\operatorname{diag}\left[l_{1}, l_{2}, \ldots, l_{n}\right]$ with $0<l_{i} \leq 1, \forall i=1,2, \ldots, n$, and

$$
\varphi(t, x(t))=\operatorname{sat}(K x(t))-L K x(t) ;
$$

then the following are equivalent:

(1) $(\operatorname{sat}(K x(t))-L K x(t))^{T}(\operatorname{sat}(K x(t))-K x(t)) \leq 0$,

(2) $\varphi(t, x(t))^{T}(\varphi(t, x(t))-(K-L K) x(t)) \leq 0$,

(3) $\|\varphi(t, x(t))\| \leq\|K-L K\|\|x(t)\|$. follows:

Then the closed-loop system (13) can be rewritten as

$$
\begin{aligned}
{ }_{0}^{C} D_{t}^{\alpha} x(t) & =\widetilde{A} x(t)+B f(x(t))+B \text { sat }(K x(t)) \\
& =A x(t)+B f(x(t))+B \varphi(t, x(t)),
\end{aligned}
$$

where $A=\widetilde{A}+B L K$.

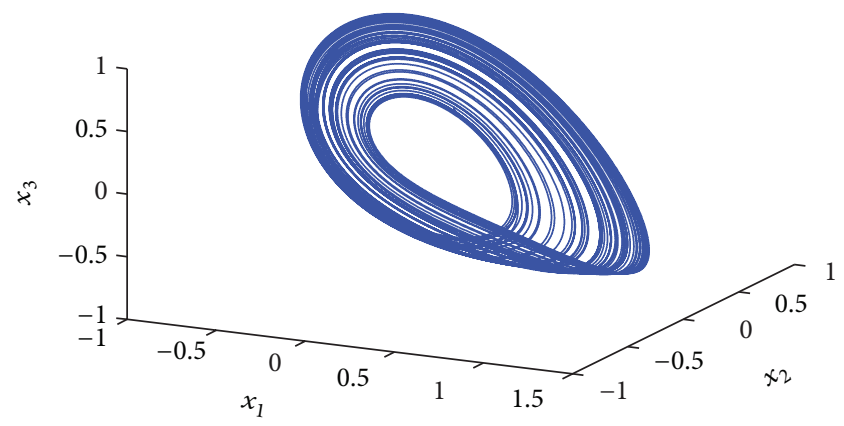

FIGURE 1: Chaotic behavior of fractional-order Genesio-Tesi system.

Theorem 13. Consider system (21). Suppose that Assumption 4 is satisfied. If we can choose matrices $L$ and $K$ appropriately such that $|\arg (\operatorname{eig}(A))|>\alpha \pi / 2$ and $\alpha\|A\|>C$, where $C>0$ will be defined later, then system (21) is asymptotically stable.

Proof. By taking the Laplace transform on system (21), we have

$$
s^{\alpha} X(s)=s^{\alpha-1} x_{0}+A X(s)+B \mathscr{L}\{f(x)\}+B \mathscr{L}\{\varphi(t, x)\},
$$

where $X(s)$ is the Laplace transform of $x(t)$. Let $I$ denote the $n \times n$ identity matrix; we can obtain

$$
X(s)=\left(I s^{\alpha}-A\right)^{-1}\left(s^{\alpha-1} x_{0}+B \mathscr{L}\{f(x)\}+B \mathscr{L}\{\varphi(t, x)\}\right) .
$$

Taking the Laplace inverse transform on (19), we have the solution of (21):

$$
\begin{aligned}
x(t)= & E_{\alpha, 1}\left(A(t)^{\alpha}\right) x_{0} \\
& +\int_{0}^{t}(t-\tau)^{\alpha-1} E_{\alpha, \alpha}\left(A(t-\tau)^{\alpha}\right) f(x) d \tau \\
& +\int_{0}^{t}(t-\tau)^{\alpha-1} E_{\alpha, \alpha}\left(A(t-\tau)^{\alpha}\right) \varphi(t, x) d \tau .
\end{aligned}
$$

According to Lemma 2, there exist some constants $c_{i}>0$, $i=1,2$ such that

$$
\begin{aligned}
\|x(t)\| \leq & \frac{c_{1}\left\|x_{0}\right\|}{1+\|A\| t^{\alpha}} \\
& +\int_{0}^{t} \frac{(t-\tau)^{\alpha-1} c_{2}}{1+\|A\|(t-\tau)^{\alpha}}\|f(x)\| d \tau \\
& +\int_{0}^{t} \frac{(t-\tau)^{\alpha-1} c_{2}}{1+\|A\|(t-\tau)^{\alpha}}\|\varphi(t, x)\| d \tau .
\end{aligned}
$$




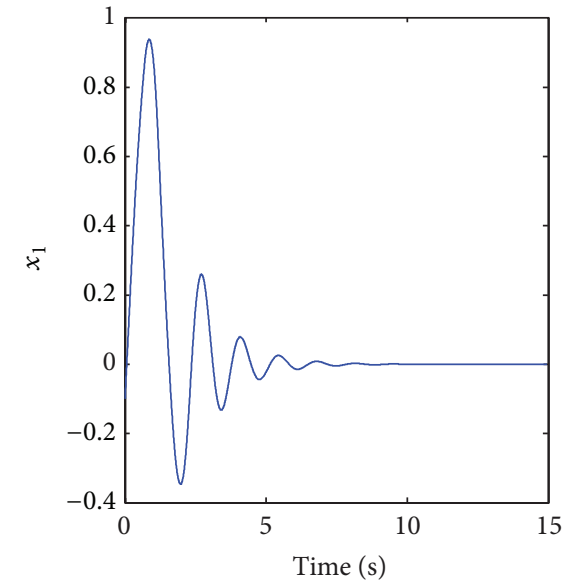

(a)

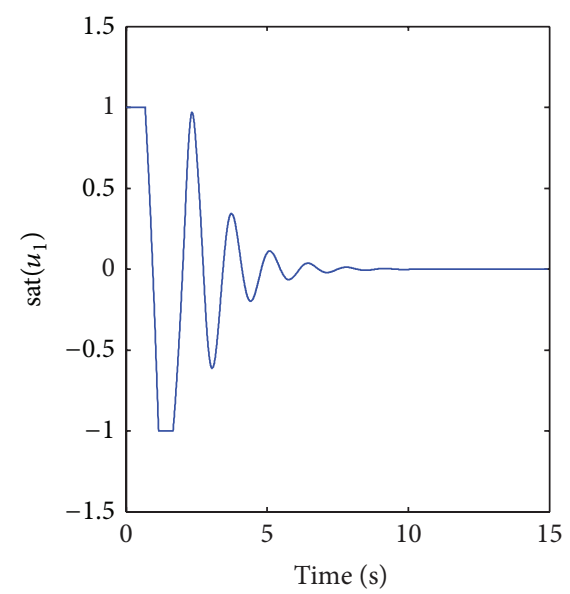

(d)

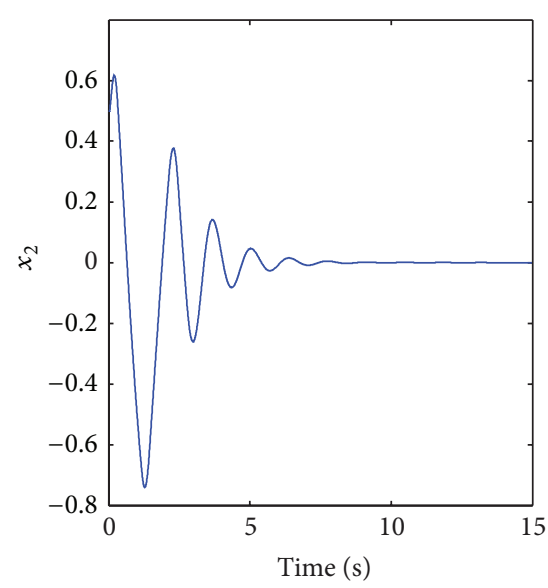

(b)

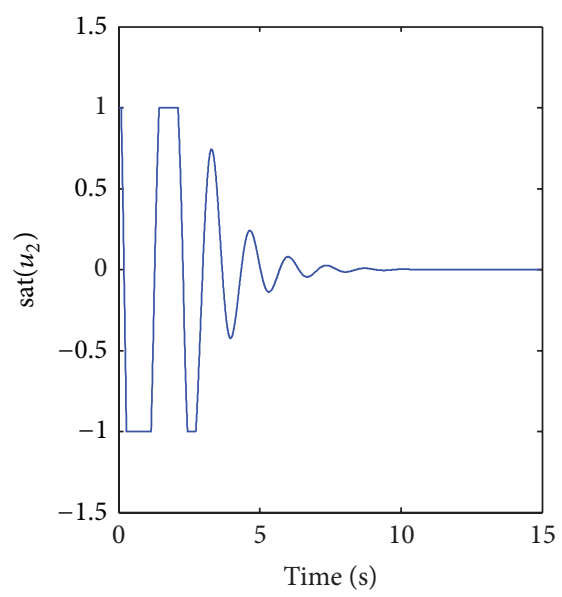

(e)

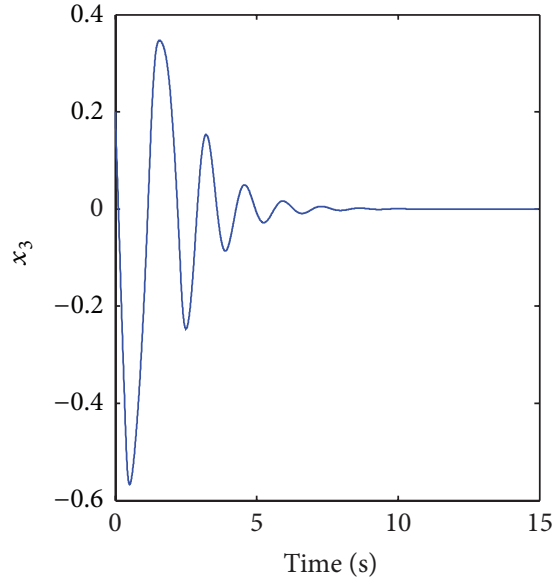

(c)

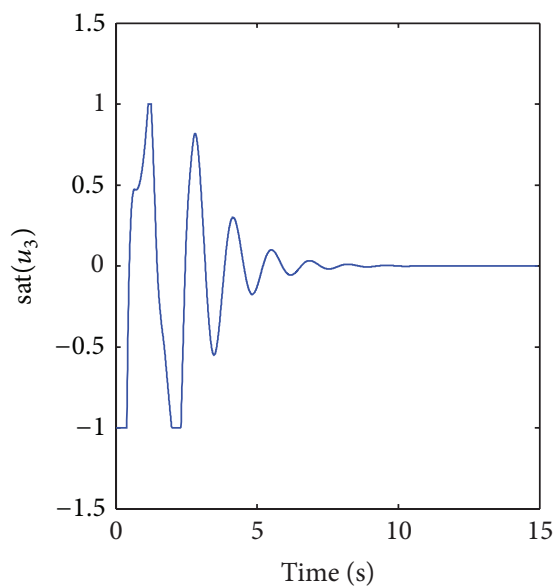

(f)

Figure 2: Simulation results for fractional-order Genesio-Tesi system. (a), (b), and (c): time response of $x_{1}, x_{2}$, and $x_{3}$; (d), (e), and (f): time response of $\operatorname{sat}\left(u_{1}\right)$, sat $\left(u_{2}\right)$, and $\operatorname{sat}\left(u_{3}\right)$.

Noting that

$$
\|f(x(t))\| \leq l\|x(t)\|,
$$

then (32) can be rewritten as

$$
\begin{aligned}
\|x(t)\| \leq & \frac{c_{1}\left\|x_{0}\right\|}{1+\|A\| t^{\alpha}} \\
& +\int_{0}^{t} \frac{(t-\tau)^{\alpha-1} c_{2} l}{1+\|A\|(t-\tau)^{\alpha}}\|x(t)\| d \tau \\
& +\int_{0}^{t} \frac{(t-\tau)^{\alpha-1} c_{2}}{1+\|A\|(t-\tau)^{\alpha}}\|\varphi(t, x)\| d \tau .
\end{aligned}
$$

From Definition 11 and Lemma 12, we know that $\varphi(t, x(t))$ satisfies

$$
\|\varphi(t, x(t))\| \leq\|K-L K\|\|x(t)\| .
$$

Let $C=c_{2} l+c_{2}\|K-L K\|$; we can obtain

$$
\|x(t)\| \leq \frac{c_{1}\left\|x_{0}\right\|}{1+\|A\| t^{\alpha}}
$$

$$
+C \int_{0}^{t} \frac{(t-\tau)^{\alpha-1}}{1+\|A\|(t-\tau)^{\alpha}}\|x(t)\| d \tau .
$$

By using Theorem 8 , if $\alpha\|A\|>C$, we have

$$
\lim _{t \rightarrow \infty}\|x(t)\|=0 \text {. }
$$

This ends the proof.

\section{Simulation Studies}

In this section, two fractional-order nonlinear (chaotic) systems are utilized to show the effectiveness of the proposed control method.

Example 14 (fractional-order Genesio-Tesi chaotic system). Consider the following. 


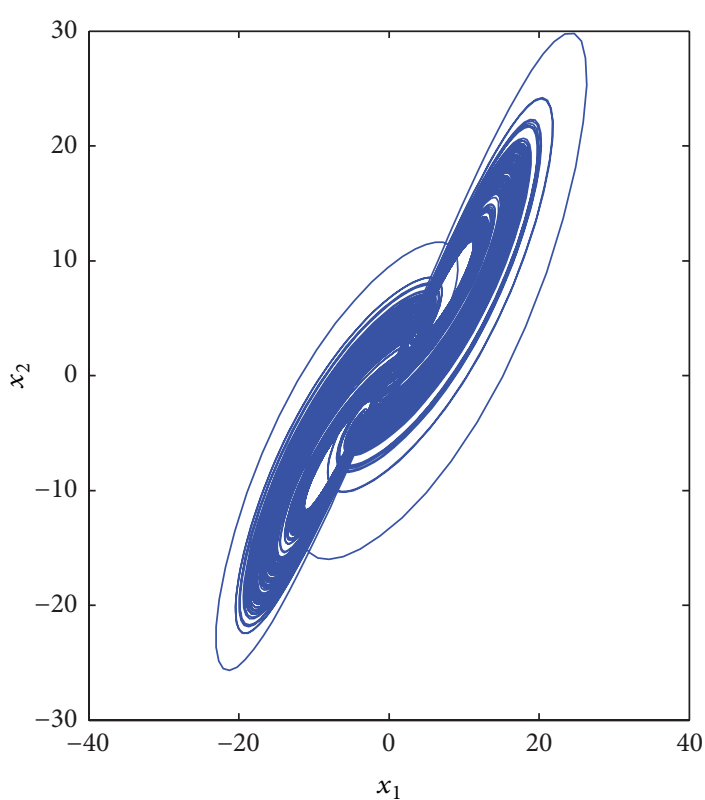

(a)

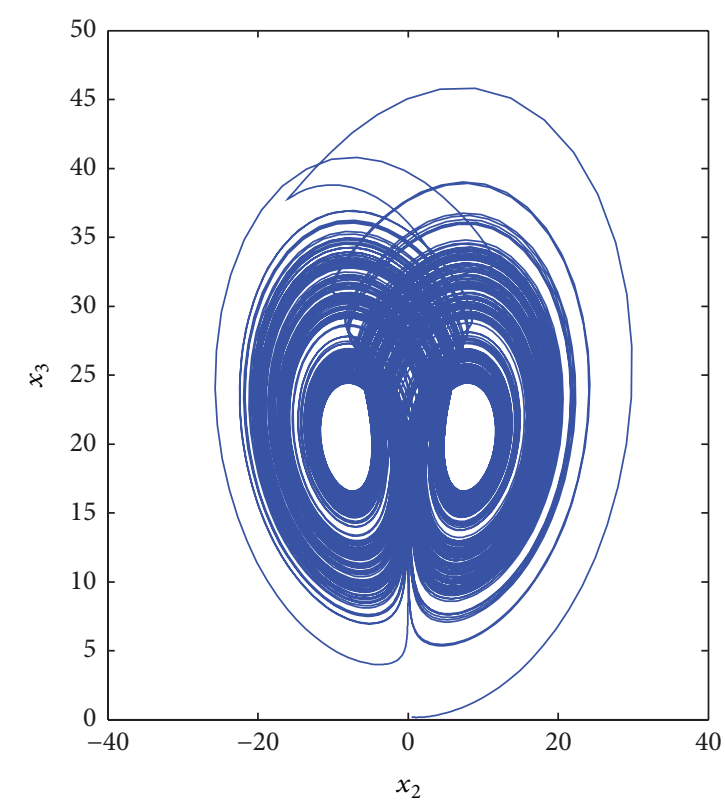

(b)

FIGURE 3: Simulation results for fractional-order Lü system. (a): $x_{1}-x_{2}$ plane; (b): $x_{2}-x_{3}$ plane.

The fractional-order Genesio-Tesi chaotic system can be written as $[23,24]$

$$
\begin{gathered}
{ }_{0}^{C} D_{t}^{\alpha} x_{1}(t)=x_{2}(t), \\
{ }_{0}^{C} D_{t}^{\alpha} x_{2}(t)=x_{3}(t), \\
{ }_{0}^{C} D_{t}^{\alpha} x_{3}(t)=-b_{1} x_{1}(t)-b_{2} x_{2}(t)-b_{3} x_{3}(t)+b_{4} x_{1}^{2}(t),
\end{gathered}
$$

where $b_{i}, i=1,2,3,4$ are system parameters. Then we have $f(x)=\left[0,0, b_{4} x_{1}^{2}(t)\right]^{T}, \widetilde{A}=\left[\begin{array}{ccc}0 & 1 & 0 \\ 0 & 0 & 1 \\ -b_{1} & -b_{2} & -b_{3}\end{array}\right]$.

And we can easily know that

$$
\lim _{x \rightarrow 0} \frac{\|f(x)\|}{\|x(t)\|} \leq \lim _{x \rightarrow 0}\left|b_{4} x_{1}(t)\right|=0,
$$

which means Assumption 4 is satisfied.

In Figure 1 the chaotic behavior of the fractional-order Genesio-Tesi chaotic system is depicted with the following parameters: $b_{1}=1.1, b_{2}=1.1, b_{3}=0.45, b_{4}=1$, the fractional order $\alpha: \alpha=0.98$, and the initial values $x(0)=-0.1, y(0)=$ $0.5, z(0)=0.2$. As we can see in the figure, the fractionalorder Genesio-Tesi system has one scroll attractor.

The control gain matrices are chosen as $L=$ $\operatorname{diag}[0.7,0.7,0.7], K=\left[\begin{array}{ccc}1.4286 & 2.8571 & 0 \\ 0 & 0 \\ -5.5714 & -4286 & 5.7143 \\ -9.3571\end{array}\right]$; then we have $A=\widetilde{A}+L K=\left[\begin{array}{ccc}1 & 3 & 0 \\ 0 & 1 & 5 \\ -5 & -6 & -7\end{array}\right]$.

From the above discussion, we know that $\|A\|=11.44$ and $|\arg (\operatorname{eig}(A))|=[3.141,1.710,-1.710]^{T}$. Then we have $|\arg (\operatorname{eig}(A))|>\alpha \pi / 2$ and $\alpha\|A\|>C$.

Let $u_{01}=u_{02}=u_{03}=1$. The simulation results can be seen in Figure 2. Form the results, we can conclude that the controlled fractional-order Genesio-Tesi system is asymptotically stable and good control performance has been achieved.

Example 15 (fractional-order Lü system). Consider the following.

The so-called Lü system is known as a bridge between Chen's system and Lorenz's system. Its fractional form can be described as follows [25]:

$$
\begin{aligned}
& { }_{0}^{C} D_{t}^{\alpha} x_{1}(t)=a\left(x_{2}(t)-x_{1}(t)\right), \\
& { }_{0}^{C} D_{t}^{\alpha} x_{2}(t)=-x_{1}(t) x_{3}(t)+c x_{2}(t), \\
& { }_{0}^{C} D_{t}^{\alpha} x_{3}(t)=-b x_{3}(t)+x_{1}(t) x_{2}(t),
\end{aligned}
$$

where $0<\alpha<1$ is derivative order and $a, b$, and $c$ are system parameters. We can rewrite the fractional Lü system as the form (9). Then we have $f(x)=\left[0,-x_{1}(t) x_{3}(t), x_{1}(t) x_{2}(t)\right]^{T}$ and $\widetilde{A}=\left[\begin{array}{ccc}-a & a & 0 \\ 0 & c & 0 \\ 0 & 0 & -b\end{array}\right]$.

Apparently, Assumption 4 is satisfied. In Figure 3 phase trajectory for fractional Lü system is depicted with derivative order $\alpha=0.95$ and parameters $a=36, b=3$, and $c=20$. The initial condition is chosen as $x(0)=x_{0}=[-0.1,0.5,0.2]^{T}$.

In the simulation, the control gain matrices are chosen as $L=\operatorname{diag}[0.95,0.92,0.99], K=\left[\begin{array}{ccc}37 & -33 & 1 \\ 2 & -19 & 5 \\ -5 & -6 & -4\end{array}\right]$; then we have $A=\widetilde{A}+L K=\left[\begin{array}{ccc}5.354 & -0.879 & 1.121 \\ 2.162 & -0.562 & 5.410 \\ -5.821 & -6.989 & -7.663\end{array}\right]$.

From the above discussion, we know that $\|A\|=12.112$ and $|\arg (\operatorname{eig}(A))|=[3.141,1.563,-1.563]^{T}$. Then we have $|\arg (\operatorname{eig}(A))|>\alpha \pi / 2$ and $\alpha\|A\|>C$.

Let $u_{01}=u_{02}=u_{03}=10$. The simulation results are shown in Figures 4 and 5. From the simulation results, we can see that the controlled fractional-order Lü system's 


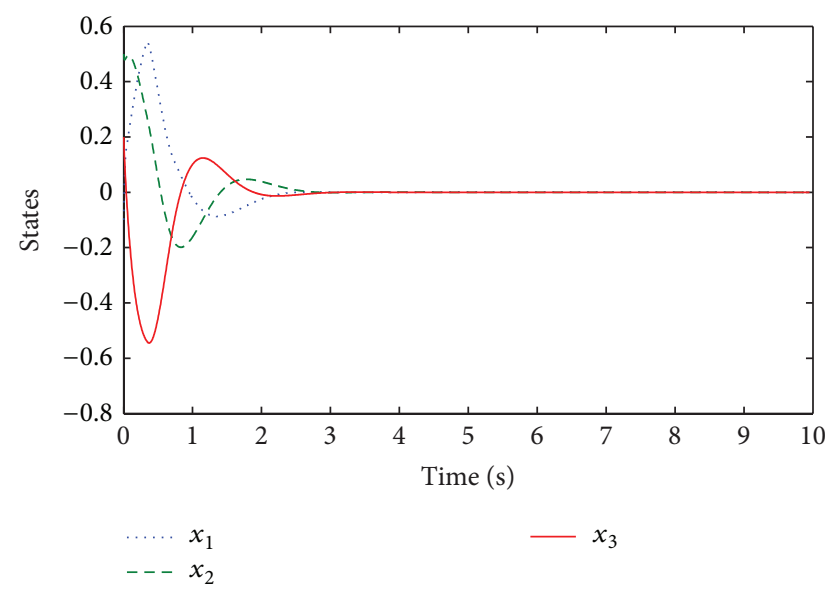

FIGURE 4: State trajectories of fractional-order Lü system.

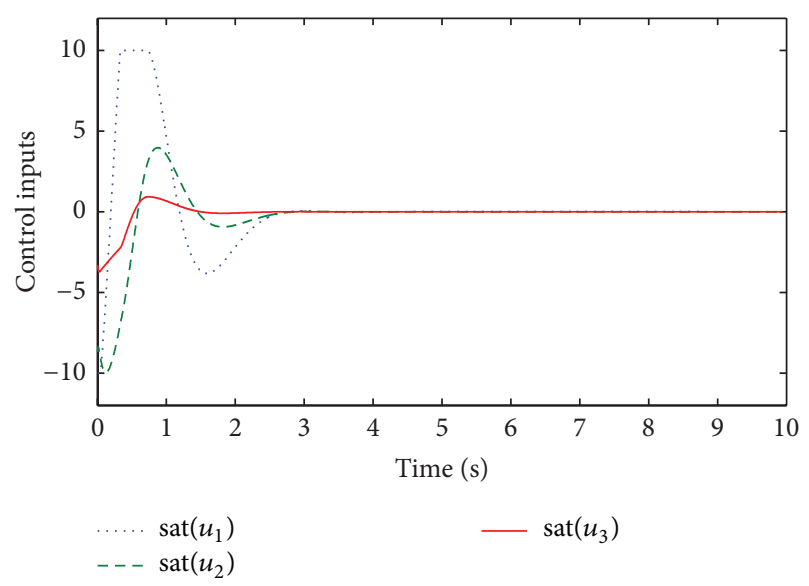

Figure 5: Time responses of control inputs subject to input saturation.

states have a fast convergence and the closed-loop system is asymptotically stable. Good control performance has been achieved. Figure 5 shows the boundedness of the control input subject to input saturation.

\section{Conclusions}

We investigate control problem for a class of fractionalorder nonlinear systems subject to input saturation by means of linear state-feedback control. Based on the sector bounded condition of the saturation function, the MittagLeffler function, and the Laplace transform technique, a sufficient condition is given for the stabilization of such systems. It is shown that linear state-feedback controller can be used to control the fractional-order nonlinear systems. Two simulation studies are given to confirm the effectiveness of the proposed method.

\section{Conflict of Interests}

The author declares that there is no conflict of interests regarding the publication of this paper.

\section{Acknowledgments}

This work is supported by National Natural Science Foundation of China (Grant no. 61001086) and Fundamental Research Funds for the Central Universities (Grant no. ZYGX2011X004).

\section{References}

[1] J. T. Machado, V. Kiryakova, and F. Mainardi, "Recent history of fractional calculus," Communications in Nonlinear Science and Numerical Simulation, vol. 16, no. 3, pp. 1140-1153, 2011.

[2] D. R. Zhu, C. X. Liu, and B. N. Yan, "Drive-response synchronization of a fractional-order hyperchaotic system and its circuit implementation," Mathematical Problems in Engineering, vol. 2013, Article ID 815765, 8 pages, 2013.

[3] S. Djennoune and M. Bettayeb, "Optimal synergetic control for fractional-order systems," Automatica, vol. 49, no. 7, pp. $2243-$ 2249, 2013.

[4] I. Podlubny, Fractional Differential Equations, vol. 198 of Mathematics in Science and Engineering, Academic Press, San Diego, Calif, USA, 1999.

[5] S. Kamal, A. Raman, and B. Bandyopadhyay, "Finite-time stabilization of fractional order uncertain chain of integrator: an integral sliding mode approach," IEEE Transactions on Automatic Control, vol. 58, no. 6, pp. 1597-1602, 2013.

[6] D. Matignon, "Stability results for fractional differential equations with applications to control processing," in Computational Engineering in Systems Applications, pp. 963-968, IMACS, IEEE-SMC, Lille, France, 1996.

[7] H.-S. Ahn and Y. Q. Chen, "Necessary and sufficient stability condition of fractional-order interval linear systems," Automatica, vol. 44, no. 11, pp. 2985-2988, 2008.

[8] J.-G. Lu and G. R. Chen, "Robust stability and stabilization of fractional-order interval systems: an LMI approach," IEEE Transactions on Automatic Control, vol. 54, no. 6, pp. 1294-1299, 2009.

[9] J.-G. Lu and Y.-Q. Chen, "Robust stability and stabilization of fractional-order interval systems with the fractional order $0<$ $\alpha<1$ case," IEEE Transactions on Automatic Control, vol. 55, no. 1, pp. 152-158, 2010.

[10] J. Sabatier, M. Moze, and C. Farges, "LMI stability conditions for fractional order systems," Computers \& Mathematics with Applications, vol. 59, no. 5, pp. 1594-1609, 2010.

[11] D. Qian, C. Li, R. P. Agarwal, and P. J. Y. Wong, "Stability analysis of fractional differential system with Riemann-Liouville derivative," Mathematical and Computer Modelling, vol. 52, no. 5-6, pp. 862-874, 2010.

[12] X.-J. Wen, Z.-M. Wu, and J.-G. Lu, "Stability analysis of a class of nonlinear fractional-order systems," IEEE Transactions on Circuits and Systems II, vol. 55, no. 11, pp. 1178-1182, 2008.

[13] Y.-H. Lim, K.-K. Oh, and H.-S. Ahn, "Stability and stabilization of fractional-order linear systems subject to input saturation," IEEE Transactions on Automatic Control, vol. 58, no. 4, pp. 1062 1067, 2013.

[14] X. F. Zhang, L. Liu, G. Feng, and Y. Z. Wang, "Asymptotical stabilization of fractional-order linear systems in triangular form," Automatica, vol. 49, no. 11, pp. 3315-3321, 2013.

[15] Y. Li, Y. Q. Chen, and I. Podlubny, "Mittag-Leffler stability of fractional order nonlinear dynamic systems," Automatica, vol. 45, no. 8, pp. 1965-1969, 2009. 
[16] Y. Li, Y. Q. Chen, and I. Podlubny, "Stability of fractionalorder nonlinear dynamic systems: Lyapunov direct method and generalized Mittag-Leffler stability," Computers \& Mathematics with Applications, vol. 59, no. 5, pp. 1810-1821, 2010.

[17] S. J. Sadati, D. Baleanu, D. A. Ranjbar, R. Ghaderi, and T. Abdeljawad, "Mittag-Leffler stability theorem for fractional nonlinear systems with delay," Abstract and Applied Analysis, vol. 2010, Article ID 108651, 7 pages, 2010.

[18] W. H. Deng, "Smoothness and stability of the solutions for nonlinear fractional differential equations," Nonlinear Analysis. Theory, Methods \& Applications, vol. 72, no. 3-4, pp. 1768-1777, 2010.

[19] L. P. Chen, Y. Chai, R. C. Wu, and J. Wang, "Stability and stabilization of a class of nonlinear fractional-order systems with Caputo derivative," IEEE Transactions on Circuits and Systems, vol. 59, no. 9, pp. 602-606, 2012.

[20] T. Gubner, M. Jost, and J. Adamy, "Controller design for a class of nonlinear systems with input saturation using convex optimization," Systems \& Control Letters, vol. 61, no. 1, pp. 258265, 2012.

[21] H. K. Khalil, Nonlinear Systems, Prentice-Hall, Englewood Cliffs, NJ, USA, 3rd edition, 2002.

[22] C. Corduneanu, Principles of Differential and Integral Equations, Allyn and Bacon, Boston, Mass, USA, 1971.

[23] K. Diethelm and N. J. Ford, "Analysis of fractional differential equations," Journal of Mathematical Analysis and Applications, vol. 265, no. 2, pp. 229-248, 2002.

[24] J. G. Lu, "Chaotic dynamics and synchronization of fractionalorder Arneodo's systems," Chaos, Solitons and Fractals, vol. 26, no. 4, pp. 1125-1133, 2005.

[25] S. Dadras and H. R. Momeni, "Control of a fractional-order economical system via sliding mode," Physica A, vol. 389, no. 12, pp. 2434-2442, 2010. 


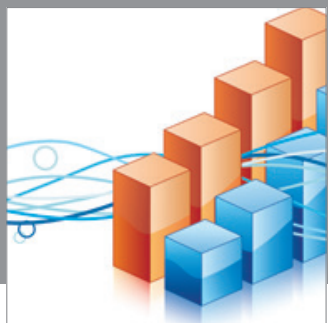

Advances in

Operations Research

mansans

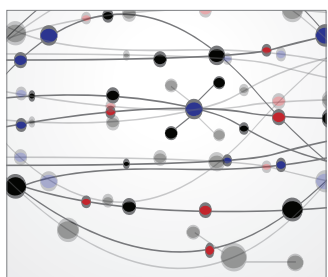

The Scientific World Journal
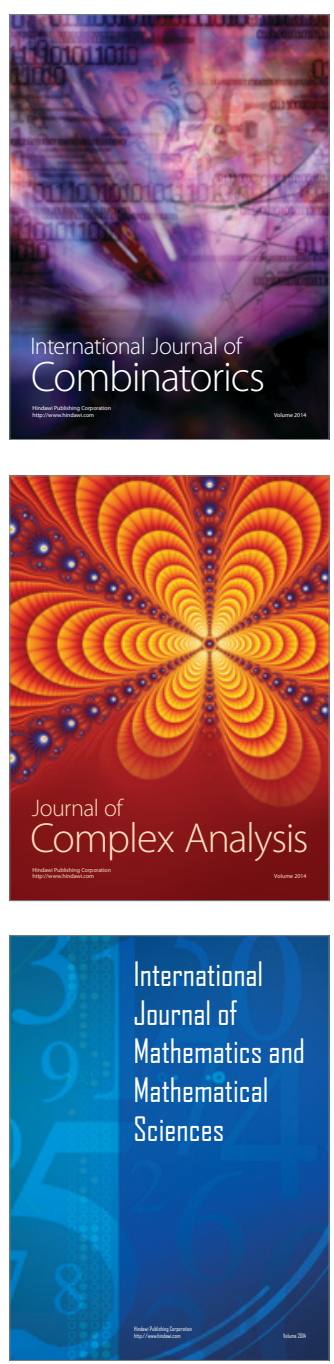
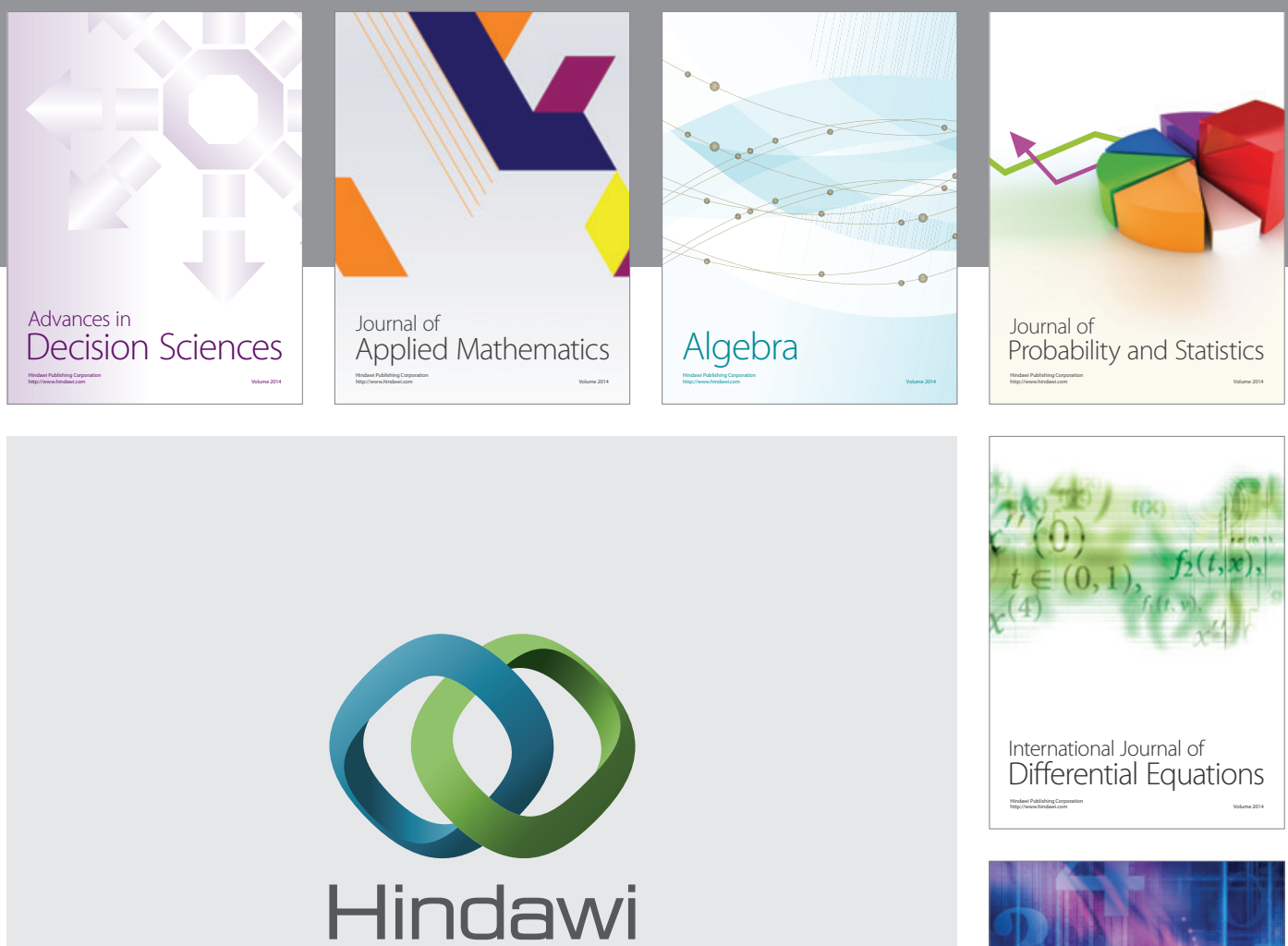

Submit your manuscripts at http://www.hindawi.com
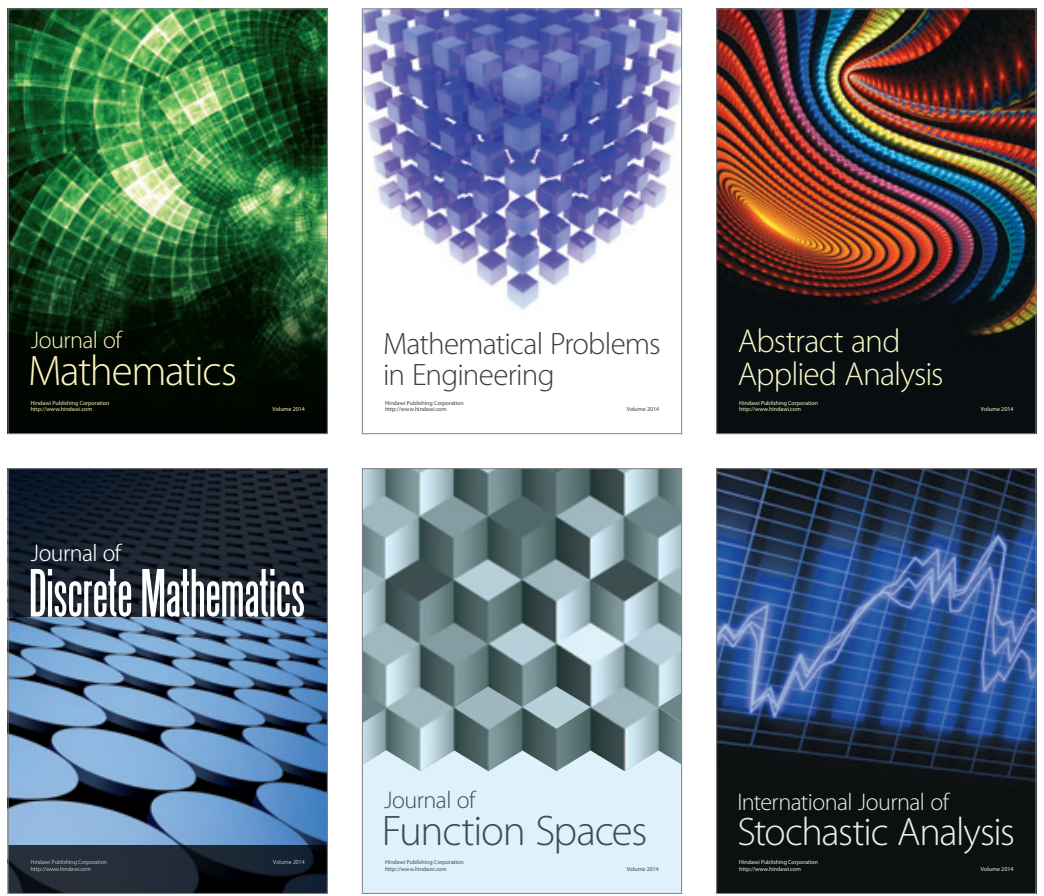

Journal of

Function Spaces

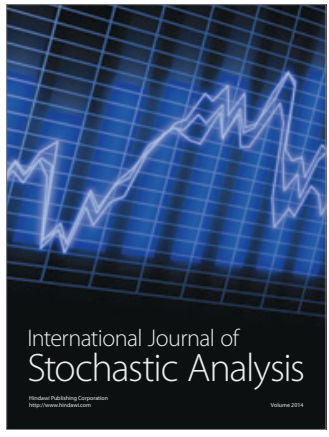

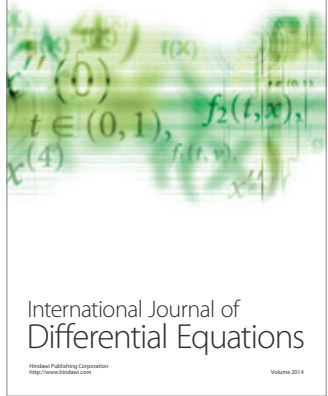
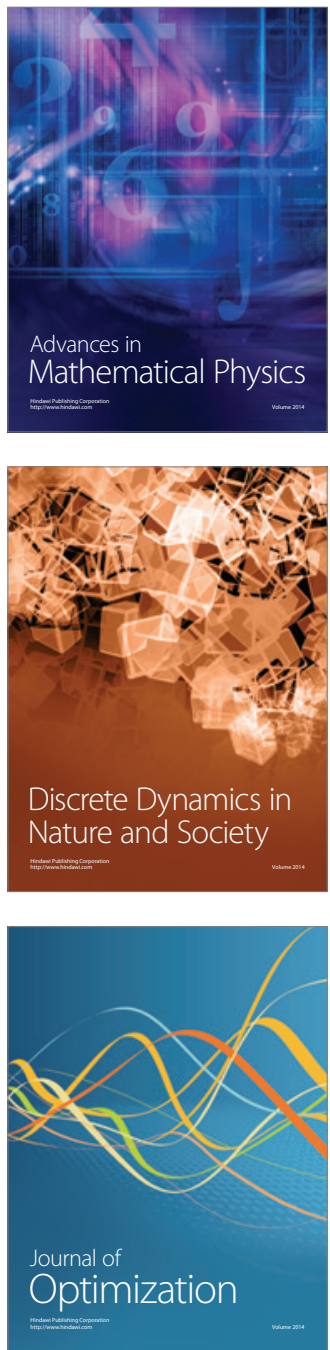